\title{
Epistatic interaction between KIR3DS1 and $H L A-B$ delays the progression to AIDS
}

\author{
Maureen P. Martin ${ }^{1 \star}$, Xiaojiang Gao ${ }^{1 \star}$, Jeong-Hee Lee ${ }^{2,9}$, George W. Nelson ${ }^{1}$, Roger Detels ${ }^{3}$, James \\ J. Goedert ${ }^{4}$, Susan Buchbinder ${ }^{5}$, Keith Hoots ${ }^{6}$, David Vlahov ${ }^{7}$, John Trowsdale ${ }^{8}$, Michael Wilson ${ }^{8,9}$, \\ Stephen J. O'Brien ${ }^{2} \&$ Mary Carrington ${ }^{1}$ \\ *These authors contributed equally to this work.
}

Published online: 22 July 2002, doi:10.1038/ng934

\begin{abstract}
Natural killer (NK) cells provide defense in the early stages of the innate immune response against viral infections by producing cytokines and causing cytotoxicity ${ }^{1}$. The killer immunoglobulin-like receptors (KIRs) on NK cells regulate the inhibition and activation of NK-cell responses through recognition of human leukocyte antigen (HLA) class I molecules on target cells ${ }^{2}$. KIR and HLA loci are both highly polymorphic, and some HLA class I products bind and trigger cell-surface receptors specified by $K I R$ genes. Here we report that the activating $K I R$ allele KIR3DS1, in combination with HLA-B alleles that encode molecules with isoleucine at position 80 (HLA-B Bw480Ile), is associated with delayed progression to AIDS in individuals infected with human immunodeficiency virus type 1 (HIV-1). In the absence of KIR3DS1, the HLA-B BW4-80lle allele was not associated with any of the AIDS outcomes measured. By contrast, in the absence of HLA-B BW4-80lle alleles, KIR3DS1 was significantly associated with more rapid progression to AIDS. These observations are strongly suggestive of a model involving an epistatic interaction between the two loci. The strongest synergistic effect of these loci was on progression to depletion of $\mathrm{CD4}^{+} \mathrm{T}$ cells, which suggests that a protective response of NK cells involving KIR3DS1 and its HLA class I ligands begins soon after HIV-1 infection.
\end{abstract}

The KIR genes, located on chromosome 19q13.4 in the leukocyte receptor complex, encode a group of receptors that are expressed on NK cells and a subset of $\mathrm{T}$ cells that recognize major histocompatibility complex (MHC) class I molecules ${ }^{1}$. NK cell-mediated cytolysis can be inhibited by the expression of appropriate HLA class I molecules on target cells, whereas activation of NK cells is associated with an absence or decreased expression of these molecules ${ }^{2}$. Several viruses escape MHC class I-restricted cytotoxic T-lymphocyte (CTL) responses by downregulating the expression of class I molecules on the infected cell surface ${ }^{3}$, which justifies the need for a defense system that responds to the absence of self class I molecules. The nef gene product of HIV-1 is known to diminish levels of HLA-A and HLA-B on infected cells, thereby mitigating recognition by $\mathrm{CTLs}^{4}$ but providing a potential stimulus for NK-cell activation.

The KIR genes are located in a segment of DNA that has undergone expansion and contraction over time, probably through unequal crossing over. Thus, KIR haplotypes vary in the number and type of genes ${ }^{5}$, although a few framework loci, such as the gene KIR3DL1, are present on all or nearly all haplotypes ${ }^{6}$. KIR3DL1 encodes receptors with three extracellular immunoglobulin-like domains - the region of the molecule that determines ligand specificity ${ }^{7}$. High-resolution genotypic analysis of families ${ }^{8}$, segregation

Table $1 \bullet$ Bw4/Bw6 motifs and their corresponding alleles

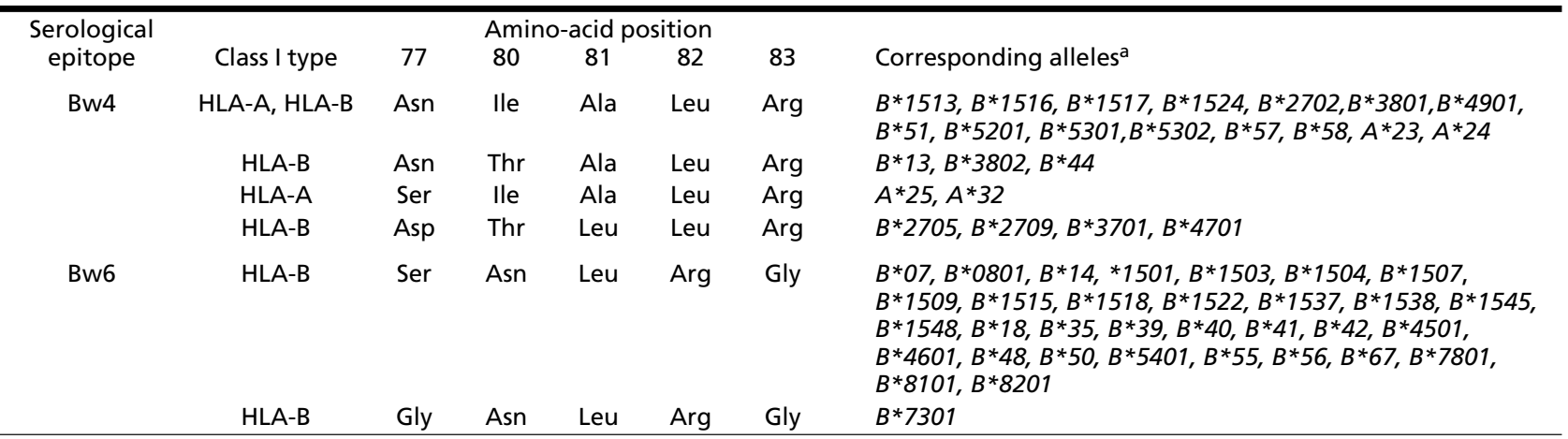

anly alleles observed in the individuals who were typed are listed (see URL in Methods).

\footnotetext{
${ }^{1}$ Basic Research Program, SAIC Frederick, and ${ }^{2}$ Laboratory of Genomic Diversity, National Cancer Institute (NCI), Frederick, Maryland 21702-1201, USA. ${ }^{3}$ Department of Epidemiology, School of Public Health, University of California, Los Angeles, California 90095, USA. ${ }^{4}$ Viral Epidemiology Branch, Division of Cancer Epidemiology and Genetics, NCI, Executive Plaza North, Bethesda, Maryland 20892, USA. ${ }^{5}$ San Francisco City Clinic Cohort, San Francisco, California 94102, USA. ${ }^{6}$ Gulf States Hemophilia Center, University of Texas Health Science Center, Houston, Texas 77030, USA. ${ }^{7}$ The Johns Hopkins School of Hygiene and Public Health, Baltimore, Maryland 21205, USA. ${ }^{8}$ University of Cambridge, Immunology Division, Department of Pathology, Tennis Court Road, Cambridge CB2 1QP, UK. ${ }^{9}$ Present addresses: Department of Pathology, College of Medicine, GyeonSang National University, Chinju, South Korea (J.-H.L.); GlaxoSmithKline, Gunnels Wood Road, Stevenage, SG1 2NY, UK (M.W). Correspondence should be addressed to M.C. (e-mail: carringt@ncifcrf.gov).
} 
analysis from 59 families from the Centre d'Etude du Polymorphisme Humain (M.C., unpublished data) and sequence analyses of KIR haplotypes ${ }^{6}$ indicate that KIR3DL1 and KIR3DS1, which were originally thought to be separate genes, segregate as alleles. Several KIR3DL1 alleles that differ by $1-21$ bp have been identified $^{8}$. We refer to this locus as KIR3DL1/3DS1.

KIR3DL1 encodes molecules with long cytoplasmic tails that inhibit NK-cell activity on ligand binding 9 . KIR3DS1 encodes molecules with short cytoplasmic tails and a charged lysine residue in the transmembrane region-the hallmarks of an activating $\mathrm{KIR}^{10,11}$. Other activating KIRs, such as KIR2DS2, mediate their activating signal through an associated transmembrane adapter called DAP12 after interaction with an appropriate ligand ${ }^{10}$. This is probably also the case for KIR3DS1, although it has not been demonstrated. Inspection of the nucleotide sequence of the allele KIR3DS1 suggests that it may have been derived from an unequal crossing-over event between exons encoding the extracellular domains of an ancestral KIR3DL1 and exons encoding the transmembrane and cytoplasmic domains of an ancestral activating $K I R$ gene.

All HLA-B molecules express one of two mutually exclusive serological epitopes, Bw4 and Bw6, that are specified by five variable amino acids spanning positions $77-83$ (Table 1$)^{12}$. Bw6 is present in about two-thirds of all HLA-B molecules and is found exclusively on HLA-B molecules, whereas Bw4 is present in the remaining third of HLA-B and in several HLA-A molecules (see URL in Methods). Receptor-ligand binding and lysis inhibition assays have shown that Bw4-containing HLA-B molecules are ligands for the NK-cell receptor KIR3DL1, and thereby inhibit the lysis of target cells expressing Bw4 (ref. 13). But some Bw4positive HLA-B types seem to be more effective than others in these assays ${ }^{7,14}$, and Bw4-positive HLA-A molecules are not lig- ands for KIR3DL1 (ref. 15), which indicates that there are additional requirements for KIR3DL1 recognition. The ligand for KIR3DS1 has not been identified, but KIR3DL1 and KIR3DS1 share about $97 \%$ amino-acid sequence similarity in their extracellular domains and they may share a similar set of ligands, as do other inhibitory and activating KIRs that have high sequence similarity in their extracellular domains ${ }^{11}$.

Functional studies have shown the importance of CTLs in controlling HIV-1, and genetic epidemiological data have supported such conclusions by indicating the strong influence of HLA class I loci on progression to AIDS ${ }^{16-19}$. It has been suggested that homozygosity with respect to Bw4-positive $H L A-B$ alleles ( $H L A$ $B B w 4)$ is associated with a slower diminution of $\mathrm{CD} 4^{+} \mathrm{T}$-cell $\operatorname{count}^{20}$, although alternative epidemiological and mechanistic interpretations for these findings are plausible ${ }^{21}$.

As several data indicate that the HLA-B locus may have a primary role in regulating outcome after HIV-1 infection, we have examined whether KIR3DL1/3DS1 acts in concert with the HLA$B$ locus to control HIV-1 in an innate response early after infection. Initially, we tested the potential effect of $H L A-B B w 4$ on AIDS progression using a model that considered all other established HLA genetic effects, as well as those involving CCR5 and CCR2 genotypes that have been previously shown to affect AIDS progression, as covariates. These included HLA class I zygosity, $B^{\star} 35-P x, B^{\star} 2705, B^{\star} 5701$ and CCR5 332 heterozygosity and dominant CCR2-64I (ref. 17).

The protective influence of $H L A-B B w 4$ on the rate of progression to AIDS was confirmed, albeit weakly, in a survival analysis of 1,039 study participants (Table 2). Bw4 molecules can be divided into two groups on the basis of whether isoleucine or threonine is present at position 80 (Bw4-80Ile and Bw4-80Thr, respectively; Table 1). Receptor-binding and lysis-inhibition data suggest that
Fig. 1 Effect of HLA-B Bw4-80lle and KIR3DS1 on AIDS progression. a-c, Kaplan-Meier survival analyses comparing the effects of $H L A-B B w 4$ $801 /$ e and KIR3DS1 separately $(a, b)$ and combined $(c)$ on progression to AIDS (1993 definition). The individuals were seroconverters of all racia groups in combined cohorts. Relative hazard (RH) and significance $(P)$ are given for Cox model analyses with the covariates described in Table $2(a, b)$ $\mathrm{RH}$ and $P$ for the combined factors $(c)$ are calculated as described in Table 2 . a, Effect of HLA-B Bw4-80lle heterozygosity (light blue) or homozygosity (blue). b, Effect of KIR3DS1 heterozygosity (pink) or homozygosity (red). $c$, Effect of the combined presence of KIR3DS1 and HLA-B BW4-80lle (purple) compared with the effects of the presence of only KIR3DS1 (red) or only $H L A-B$ Bw4-80lle (blue). In a-c, gray represents individuals who lacked the factors in question. $\boldsymbol{d}$, Diseasecategory analyses comparing the frequency of KIR3DS1+HLA-B $B w 4-80 / l e$ (one or more copies of each) genotypes in rapid and slow progressors to AIDS 1993. The individuals were European American seroconverters and seroprevalents in combined cohorts. Only seroconverters who progressed to the defined end points were considered in the first three (rapid) groups. For the slow groups, all seroconverters and seroprevalents who progressed to the defined end points or had their last known follow up or censorship in the specified time were included. The number of individuals in each category is shown above the bars.

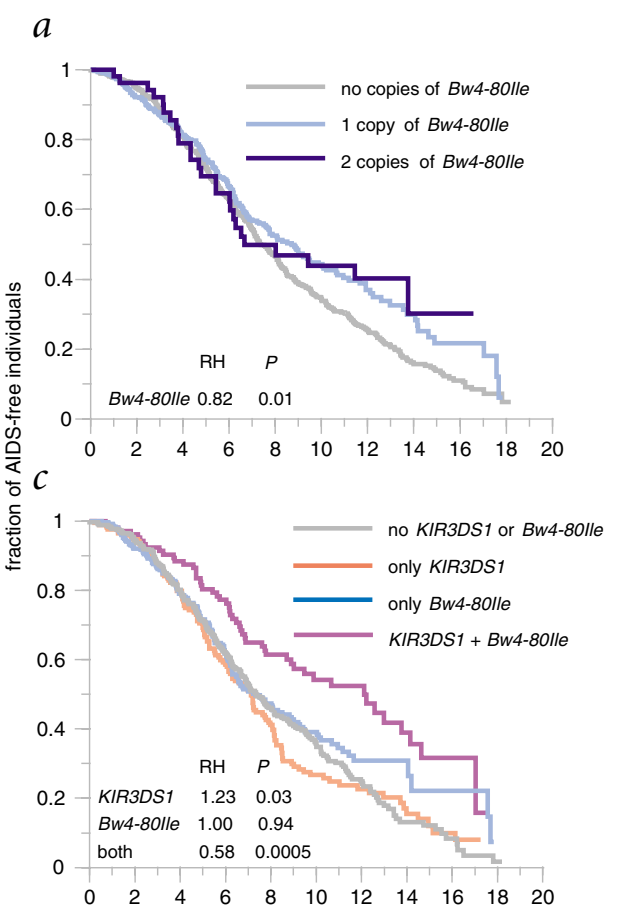

\section{$b$}

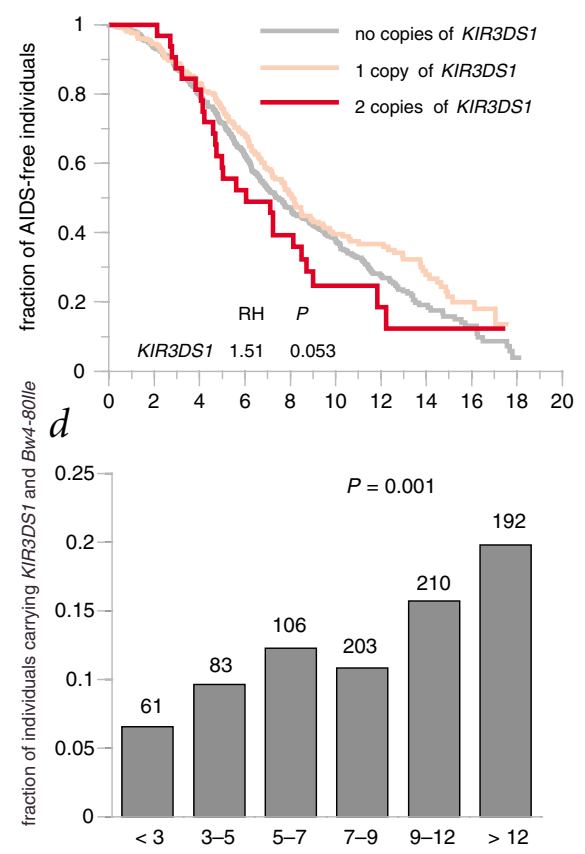

time since seroconversion $(y)$ 
HLA-B molecules containing Bw4-80Ile may be more effective ligands for KIR3DL1 than are those containing Bw4$80 \mathrm{Thr}^{7,14}$, whereas neither HLA-A Bw4 nor HLA-B Bw6 molecules bind to KIR3DL1 (refs 13,15). In addition, residue 80 of HLA-C is crucial in determining binding specificity for receptors containing two immunoglobulin domains, as shown by cytotoxicity assays ${ }^{22}$ and X-ray crystallography ${ }^{23}$. We therefore grouped $H L A-B B w 4$ alleles dichotomously according to amino-acid variation at position 80 and determined the effect of these two groups on AIDS progression.

A comparison of study participants with HLA-B Bw4-80Ile against those without these alleles indicated that the protective effect of $H L A-B \quad B w 4$ against AIDS progression could be attributed to the Bw4-80Ile genotype (Fig. $1 a$ and Table 2). No influence of the Bw4-80Thr allele was observed (Table 2).

HLA-A Bw4 alleles, all of which encode isoleucine at position 80 , had no detectable effect on AIDS progression (data not shown).

We examined the association of HLA-B Bw4-80Ile with protection against AIDS progression in the context of the KIR3DL1 and KIR3DS1 subtypes. First, we compared the effect of the KIR3DL1 and KIR3DS1 alleles on progression to AIDS in all individuals without considering the influence of $H L A-B$ Bw4-80Ile. In survival analyses of combined cohorts, a modest recessive influence of KIR3DS1 leading to more rapid AIDS progression was observed consistently (relative hazard $(\mathrm{RH})=1.31-1.86$ for different AIDS end points; Fig. $1 b$ and Table 2). This accelerating influence was apparent in Cox model analyses in which the other known AIDS restriction genotypes (HLA zygosity, $B^{\star} 27, B^{\star} 57, B^{\star} 35-P x$, CCR5$\triangle 32$ and CCR2-64I) were considered as covariates; these analyses indicated an independent recessive effect of the KIR3DS1 gene.

To explore the possible interaction between the observed effects of HLA-B Bw4-80Ile and those of KIR3DS1, we carried out survival analyses to determine the effects of $H L A-B$ Bw4-80Ile in the absence of KIR3DS1, KIR3DS1 in the absence of HLA-B Bw4-80Ile, and the two factors combined. We used the three genotypes as covariates, along with the five confounding covariates described above, in the multivariate Cox model regression. The combination of KIR3DS1 and HLA-B Bw4-80Ile was highly protective with respect to several AIDS end points, suggesting that there is an
Table 3 - Synergistic effect of KIR3DS1 and HLA-B Bw4-80Ile on progression to AIDS-related end points

\begin{tabular}{lcccc}
\hline Genetic variable & AIDS outcome & No. of individuals & $\mathrm{RH}^{\mathrm{a}}$ & $P$ value \\
KIR3DS1 & CD4 < 200 & 904 & 1.26 & 0.02 \\
& AIDS 1993 & 907 & 1.23 & 0.03 \\
& AIDS 1987 & 917 & 1.14 & 0.26 \\
Bw4-80lle & death & 916 & 1.36 & 0.01 \\
& CD4 $<200$ & 904 & 1.05 & 0.66 \\
& AIDS 1993 & 907 & 1.00 & 0.94 \\
KIR3DS1 + Bw4-80lle & AIDS 1987 & 917 & 0.92 & 0.50 \\
& death & 916 & 0.95 & 0.70 \\
& CD4 < 200 & 904 & 0.58 & 0.001 \\
& AIDS 1993 & 907 & 0.58 & 0.0005 \\
& AIDS 1987 & 917 & 0.74 & 0.10 \\
& death & 916 & 0.65 & 0.04
\end{tabular}

aResults of a single Cox model survival analysis with three codominant explanatory variables: (i) the individual's number of KIR3DS1 alleles, (ii) the number of HLA-B BW4-80lle alleles and (iii) the combination of KIR3DS1 and HLA-B BW4-80Ile, calculated as the product of the first two variables. Other factors relating to $H L A$ and $C C R$ alleles were considered as confounding covariates as described in Table 2. Alternative analyses were also carried out considering the effects of KIR3DS1 and HLA-B BW4-80Ile as dominant, both separately and in combination (see Web Table B online). 


\begin{tabular}{|c|c|c|c|c|c|c|c|}
\hline \multirow[b]{2}{*}{ Genetic variable } & \multirow[b]{2}{*}{ AIDS outcome } & \multicolumn{3}{|c|}{ European Americans } & \multicolumn{3}{|c|}{ African Americans } \\
\hline & & $\begin{array}{c}\text { No. of } \\
\text { individuals }\end{array}$ & $\mathrm{RH}$ & $P$ value & $\begin{array}{c}\text { No. of } \\
\text { individuals }\end{array}$ & $\mathrm{RH}$ & $P$ value \\
\hline KIR3DS1 & $\begin{array}{c}\text { CD4 < } 200 \\
\text { AIDS } 1993 \\
\text { AIDS } 1987 \\
\text { death }\end{array}$ & $\begin{array}{l}631 \\
633 \\
640 \\
639\end{array}$ & $\begin{array}{l}1.21 \\
1.21 \\
1.12 \\
1.34\end{array}$ & $\begin{array}{l}0.07 \\
0.06 \\
0.33 \\
0.02\end{array}$ & $\begin{array}{l}234 \\
235 \\
238 \\
238\end{array}$ & $\begin{array}{l}2.66 \\
2.23 \\
0.50 \\
0.69\end{array}$ & $\begin{array}{l}0.02 \\
0.05 \\
0.46 \\
0.74\end{array}$ \\
\hline Bw4-80Ile & $\begin{array}{c}\text { CD4 < } 200 \\
\text { AIDS } 1993 \\
\text { AIDS } 1987 \\
\text { death }\end{array}$ & $\begin{array}{l}631 \\
633 \\
640 \\
639\end{array}$ & $\begin{array}{l}1.02 \\
0.93 \\
0.80 \\
0.84\end{array}$ & $\begin{array}{l}0.87 \\
0.55 \\
0.16 \\
0.29\end{array}$ & $\begin{array}{l}234 \\
235 \\
238 \\
238\end{array}$ & $\begin{array}{l}1.20 \\
1.33 \\
1.37 \\
1.43\end{array}$ & $\begin{array}{l}0.40 \\
0.12 \\
0.20 \\
0.27\end{array}$ \\
\hline KIR3DS1 + Bw4-80Ile & $\begin{array}{c}\text { CD4 < } 200 \\
\text { AIDS } 1993 \\
\text { AIDS } 1987 \\
\text { death }\end{array}$ & $\begin{array}{l}631 \\
633 \\
640 \\
639\end{array}$ & $\begin{array}{l}0.60 \\
0.59 \\
0.78 \\
0.70\end{array}$ & $\begin{array}{l}0.006 \\
0.003 \\
0.23 \\
0.11\end{array}$ & $\begin{array}{l}234 \\
235 \\
238 \\
238\end{array}$ & $\begin{array}{l}0.18 \\
0.25 \\
1.76 \\
1.52\end{array}$ & $\begin{array}{l}0.01 \\
0.03 \\
0.59 \\
0.74\end{array}$ \\
\hline
\end{tabular}

The KIR3DS1 allele seems to influence two opposing mechanisms of viral control: one for AIDS susceptibility (KIR3DS1 homozygosity in the absence of HLA-B Bw4-80Ile) and the other for protection (KIR3DS1 + HLA-B Bw4-80Ile). The protective effect of KIR3DS1 + HLA-B Bw4-80Ile seems to be dominant over susceptibility conferred by KIR3DS1 homozygosity, because the ten study participants who were homozygous for KIR3DS1 and positive for $H L A-B$ Bw4-80Ile progressed slowly to AIDS (1993 definition; approaching acclelerating progression to AIDS is enhanced when the epistatic interaction between KIR3DS1 + HLA-B Bw4-80Ile is considered as a covariate (Table 3), which confirms the observed effect of KIR3DS1 in analyses not adjusted for combinatorial effects (Fig. 1c and Table 2). That KIR3DS1 exerts a modest accelerating influence makes the synergistic influence of KIR3DS1 + HLA-B Bw4-80Ile in slowing AIDS progression particularly notable.

Protection against AIDS conferred by the epistatic KIR3DS1+ HLA-B Bw4-80Ile genotype was apparent in Caucasian and African American cohorts (Table 4) and in combined ethnic groups (Table 3). Consistent RHs, albeit not always statistically significant, were observed in survival analyses of separate risk groups and cohorts (see Web Table A online). A categorical analysis of rapid versus slow progressors, which included several seroprevalent study participants in addition to seroconverters, also showed consistently elevated frequencies of the protective dual genotype (KIR3DS1 + HLA-B Bw4-80Ile) in slow progressors to AIDS (Fig. $1 d$ and Web Fig. A online). Thus, replication in groups stratified by ethnicity, risk (parenteral or mucosal infection) and cohort consistently support epistatic protection by the KIR3DS1 + $H L A-B B w 4-80 I l e$ genotype against rapid AIDS progression.

The synergistic effect of KIR3DS1 and HLA-B Bw4-80Ile was strongest in outcomes that took into account CD4 ${ }^{+} \mathrm{T}$-cell counts of less than 200 per $\mathrm{mm}^{3}$ (CD4 < 200 and AIDS 1993 definition; Table 3). No combinatorial effect of HLA-A Bw4-80Ile alleles with KIR3DS1 was observed $(P>0.19)$, which emphasizes the specificity of the observed effect for $H L A-B$ as opposed to other class I loci. Four subtypes of the Bw4 epitope, three of which are found in HLA-B molecules, were observed among the samples typed in this study (Table 1). Among HLA-B Bw4 molecules, positions 77, 80 and 81 vary by two or three amino acids at each site and are conserved at the remaining four positions of the epitope. Cox model analysis of each variant at positions 77 and 81 indicated that they are not associated with the protective effect observed between KIR3DS1 and HLA-B Bw4-80Ile. Together, the data suggest that HLA-B Bw4-80Ile molecules behave as ligands for KIR3DS1 and that HIV-1-infected cells expressing HLA-B Bw4-80Ile may be prone to NK-cell activity that is regulated in part by ligand binding to KIR3DS1 expressed on the NK-cell surface.

Some KIRs are expressed on a subset of T cells with a memory phenotype ${ }^{24}$, which suggests that they may regulate T-cell as well as NK-cell activity. Indeed, masking of inhibitory NK receptors on CTLs from HIV-infected individuals by monoclonal antibody has been shown to increase HIV-specific CTL activity ${ }^{25}$. It will therefore be important to determine whether our observations reflect a functional interaction of KIR3DS1 and HLA-B Bw480Ile on T cells, on NK cells, or on both. statistical significance, $\mathrm{RH}=0.43, P=0.09)$. The protective effect of heterozygous KIR3DS1/3DL1 + HLA-B Bw4-80Ile cannot be readily attributed to $K I R 3 D L 1$, because the presence of two copies of KIR3DL1 along with HLA-B Bw4-80Ile did not confer a protective effect. It is possible, however, that KIR3DS1 is not directly responsible for the observed protection, but is only a marker of a haplotype that contains the actual disease locus. If so, then this operative locus would still have to interact with $H L A-B$ $B w 4-80$ Ile or with a locus in linkage disequilibrium with HLA-B $B w 4-80 I l e$. This remains a possibility, because both KIR3DS1 and $H L A-B$ are located in genomic segments that contain other related genes involved in the immune response.

Data indicating a primary role for the $H L A-B$ locus in AIDS progression and the receptor-ligand relationship of HLA-B molecules with KIR3DL1 lend credence, however, to a model in which KIR3DS1 binds HLA-B molecules containing Bw4-80Ile, leading to activation of NK cells, T cells, or both and elimination of HIV-1-infected cells. Such events may occur before the generation of HIV-specific CTLs and continue in concert with CTLs once an acquired immune response has been initiated. A swift nonspecific response to viral infection mediated through KIR molecules would correlate well with data showing that rapid immune responses after viral exposure are beneficial in controlling the virus ${ }^{26}$. Theoretically, this same genetic combination should be protective against other viral infections, as there is no reason to suppose that the response is specific to HIV.

The recessive susceptibility effect of KIR3DS1 (in the absence of HLA-B Bw4-80Ile) may be due to other genes in linkage disequilibrium with KIR3DS1 or, alternatively, to a relatively weak dominant-protective effect of KIR3DL1 that appears neutral in comparison to the strong protective effect of KIR3DS1 + HLA-B $B w 4-80 I l e$. Differential binding of a specific antibody against KIR3DL1 to NK cells carrying various KIR3DL1 alleles ${ }^{8}$ raises the possibility that allelic variation at this locus might affect expression of the molecule; KIR3DL1 subtyping may provide some clues to potential effects of this group of alleles.

Survival analyses of KIR3DS1 with individual HLA-B Bw480Ile alleles suggested that no single allele or minor subset of $H L A-B$ Bw4-80Ile alleles could account for the observed protective effect of KIR3DS1 + HLA-B Bw4-80Ile as a whole, although the power to detect such effects was diminished. But protection by the $B w 4-80 I l e$-positive $H L A-B^{\star} 5701$ allele was evident in the presence $(\mathrm{CD} 4<200, \mathrm{RH}=0.37, P=0.05)$ or absence $(\mathrm{CD} 4<$ $200, \mathrm{RH}=0.52, P=0.008)$ of KIR3DS1, which suggests that the strong protection conferred by this allele is not completely dependent on KIR3DS1. Similarly, $H L A-B^{\star} 27$ protection is probably independent of a KIR3DS1 interaction because the most 
common $B^{\star} 27$ allele in our cohorts, $B^{\star} 2705$ ( $83 \%$ of all $B^{\star} 27$ alleles), contains Bw4-80Thr - the Bw4 motif that does not confer protection in the presence of KIR3DS1. Although we cannot rule out the possibility of KIR involvement in $B^{\star} 27$ protection, the ability of $B^{\star} 27$ molecules to present a highly conserved immunodominant HIV-1 epitope-one that may require two mutations to escape CTL recognition and still maintain viral fitness ${ }^{27}$-may be at least partly responsible for its observed protective effect.

Because of the extensive polymorphism at the HLA class I loci, strategies for grouping alleles according to similarity in specificity for peptide motifs can enhance the power and information content of HLA-disease association studies ${ }^{19,28}$. Peptides differing at positions 7 and 8 , both of which are non-anchor residues, can sometimes affect recognition of HLA class I ligands by specific KIR molecules ${ }^{29}$. But it is unlikely that the epistatic effect of KIR3DS1 + HLA-B Bw4-80Ile can be explained by peptide preference of the various HLA-B Bw480Ile molecules, because members of this group differ markedly in their preference for peptides.

The interaction between common alleles at polymorphic loci in disease resistance, as shown here for KIR3DS1 and HLA-B $B w 4-80 I l e$ in HIV-1 infection, represents a form of genetic epistasis that is distinct from cases where mutations in two or more different genes result in a disease phenotype ${ }^{30}$. Although inherently difficult to detect, the synergistic interaction between alleles at unlinked loci may be common in complex disorders such as infectious diseases. Identification of such interactions may provide new approaches to therapeutic and vaccine development.

\section{Methods}

Subjects. Individuals infected with HIV-1 for whom dates of seroconversion were known were derived from five cohorts: the Multicenter AIDS Cohort study (MACS), the Multicenter Hemophilia Cohort Study (MHCS), the Hemophilia Growth and Development Study (HGDS), the San Francisco City Clinic Cohort (SFCCC) and the AIDS Linked to Intravenous Experience (ALIVE) Study. Seroconverters from the MACS and ALIVE cohorts were representative of all HIV-infected individuals, whereas individuals from the SFCCC and MHCS cohorts showed a moderate survival bias because biological samples were unavailable for individuals with the most rapid rates of progression to AIDS. The study was approved by the Protocol Review Office of the NCI institutional review board. Informed consent was obtained at the study sites from all individuals.

HLA class I typing. We amplified genomic DNA using locus-specific primers that flanked exons 2 and 3. The PCR products were blotted on nylon membranes and hybridized with sequence-specific oligonucleotide (SSO) probes. We assigned alleles by the reaction patterns of the SSO probes. Ambiguous SSO probe typing results were resolved by sequencing analysis as described ${ }^{19}$.

KIR genotyping. Genomic DNA was typed for presence or absence of KIR3DL1 and KIR3DS1 by PCR with sequence-specific priming (PCR-SSP). We carried out PCR amplification with two pairs of specific primers for each locus. Internal control primers for a fragment of $796 \mathrm{bp}$ of the third intron of DRB1 were also included in each PCR. We amplified $20-50 \mathrm{ng}$ DNA in a volume of $20 \mu \mathrm{l}$ containing $200 \mu \mathrm{M} \mathrm{dNTP}, 100-500 \mathrm{nM}$ of specific primer, $100 \mathrm{nM}$ of internal control primer, $2 \mathrm{mM} \mathrm{MgCl}$ $67 \mathrm{mM}$ Tris, $16.6 \mathrm{mM}\left(\mathrm{NH}_{4}\right)_{2} \mathrm{SO}_{4}$, and $0.5 \mathrm{U}$ of Taq polymerase. Cycling was carried out in a GeneAmp PCR system 9700 thermal cycler (PE Applied Biosystems) as follows: $1 \mathrm{~min}$ at $96^{\circ} \mathrm{C} ; 5$ cycles of $96^{\circ} \mathrm{C}$ for $25 \mathrm{~s}$, $65^{\circ} \mathrm{C}$ for $45 \mathrm{~s}, 72^{\circ} \mathrm{C}$ for $30 \mathrm{~s} ; 21$ cycles of $96^{\circ} \mathrm{C}$ for $25 \mathrm{~s}, 60^{\circ} \mathrm{C}$ for $45 \mathrm{~s}, 72^{\circ} \mathrm{C}$ for $30 \mathrm{~s} ; 5$ cycles of $96^{\circ} \mathrm{C}$ for $25 \mathrm{~s}, 55^{\circ} \mathrm{C}$ for $1 \mathrm{~min}, 72^{\circ} \mathrm{C}$ for $2 \mathrm{~min}$; and a final extension step of $10 \mathrm{~min}$ at $72^{\circ} \mathrm{C}$. We separated PCR products in $1.5 \%$ agarose gels containing ethidium bromide and visualized the products under ultraviolet light. Primer sequences are available from the authors on request.

Statistical analysis. Survival analyses were carried out on seroconverters from all of the cohorts combined and included all individuals without regard to racial group. Four AIDS-related outcomes were considered as end points of survival analysis: a CD4 $4^{+}$T-lymphocyte count of $<200$ per $\mathrm{mm}^{3}$, progression to AIDS according to the 1993 definition of the US Centers for Disease Control (CDC), progression to AIDS according to the more stringent 1987 CDC definition, and death. We carried out Kaplan-Meier and Cox model analyses using the LIFETEST and PHREG procedures of the SAS system (SAS Institute). Genetic factors with a confirmed effect on progression to AIDS (protective genotypes of the chemokine receptors $C C R 2$ and CCR5, HLA alleles $B^{\star} 27, B^{\star} 57$ and alleles of the $B^{*} 35 P x$ group, and overall homozygosity at the HLA-A, HLA-B and $H L A-C$ loci) were included as covariates in Cox model analyses. Subjects in the Cox model analyses were stratified by race and age at seroconversion.

URL. HLA class I and II sequence alignments, http://www.anthonynolan. org.uk/HIG/data.html.

Note: Supplementary information is available on the Nature Genetics website.

\section{Acknowledgments}

We thank P. Parham for comments. This work was supported by funds from the US National Cancer Institute and National Institutes of Health. The content of this publication does not necessarily reflect the views or policies of the Department of Health and Human Services, nor does the mention of trade names, commercial products or organizations imply endorsement by the US Government.

\section{Competing interests}

The authors declare that they have no competing financial interests.

Received 16 April; accepted 17 June 2002.

1. Lanier, L.L. NK cell receptors. Annu. Rev. Immunol. 16, 359-393 (1998).

. Long, E.O. \& Rajagopalan, S. HLA class I recognition by killer cell Ig-like receptors. Semin. Immunol. 12, 101-108 (2000).

3. Lorenzo, M.E., Ploegh, H.L. \& Tirabassi, R.S. Viral immune evasion strategies and the underlying cell biology. Semin. Immunol. 13, 1-9 (2001)

4. Collins, K.L., Chen, B.K., Kalams, S.A., Walker, B.D. \& Baltimore, D. HIV-1 Nef protein protects infected primary cells against killing by cytotoxic T lymphocytes. Nature 391, 397-401 (1998).

5. Trowsdale, J. et al. The genomic context of natural killer receptor extended gene families. Immunol. Rev. 181, 20-38 (2001)

6. Wilson, M.J. et al. Plasticity in the organization and sequences of human KIR/ILT gene families. Proc. Natl Acad. Sci. USA 97, 4778-4783 (2000).

7. Rojo, S., Wagtmann, N. \& Long, E.O. Binding of a soluble p70 killer cell inhibitory receptor to $H L A-B * 5101$ : requirement for all three p70 immunoglobulin domains. Eur. J. Immunol. 27, 568-571 (1997).

8. Gardiner, C.M. et al. Different NK cell surface phenotypes defined by the DX9 antibody are due to KIR3DL1 gene polymorphism. J. Immunol. 166, 2992-3001 (2001).

9. Long, E.O. Regulation of immune responses through inhibitory receptors. Annu. Rev. Immunol. 17, 875-904 (1999).

10. Lanier, L.L., Corliss, B.C., Wu, J., Leong, C. \& Phillips, J.H. Immunoreceptor DAP12 bearing a tyrosine-based activation motif is involved in activating NK cells. Nature 391, 703-707 (1998).

11. Biassoni, R. et al. The human leukocyte antigen (HLA)-C-specific 'activatory' or 'inhibitory' natural killer cell receptors display highly homologous extracellular domains but differ in their transmembrane and intracytoplasmic portions. J. Exp. Med. 183, 645-650 (1996)

12. Muller, C.A. et al. Genetic and serological heterogeneity of the supertypic HLA-B locus specificities Bw4 and Bw6. Immunogenetics 30, 200-207 (1989).

13. Gumperz, J.E., Litwin, V., Phillips, J.H., Lanier, L.L. \& Parham, P. The Bw4 public epitope of HLA-B molecules confers reactivity with natural killer cell clones that express NKB1, a putative HLA receptor J. Exp. Med. 181, 1133-1144 (1995).

14. Cella, M., Longo, A., Ferrara, G.B., Strominger, J.L. \& Colonna, M. NK3-specific natural killer cells are selectively inhibited by Bw4- positive HLA alleles with isoleucine 80. J. Exp. Med. 180, 1235-1242 (1994).

15. Gumperz, J.E. et al. Conserved and variable residues within the Bw4 motif of HLA-B make separable contributions to recognition by the NKB1 killer cellinhibitory receptor. J. Immunol. 158, 5237-5241 (1997).

16. McMichael, A.J. \& Rowland-Jones, S.L. Cellular immune responses to HIV. Nature 410, 980-987 (2001).

17. Carrington, M., Nelson, G. \& O'Brien, S.J. Considering genetic profiles in functional studies of immune responsiveness to HIV-1. Immunol. Lett. 79, $131-140$ (2001).

18. Kaslow, R.A. et al. Influence of combinations of human major histocompatibility complex genes on the course of HIV-1 infection. Nature Med. 2, 405-411 (1996).

19. Gao, X. et al. Effect of a single amino acid change in MHC class I molecules on the rate of progression to AIDS. N. Engl. J. Med. 344, 1668-1675 (2001).

20. Flores-Villanueva, P.O. et al. Control of HIV-1 viremia and protection from AIDS are associated with HLA-Bw4 homozygosity. Proc. Natl Acad. Sci. USA 98, 5140-5145 (2001). 
21. O'Brien, S.J., Gao, X. \& Carrington, M. HLA and AIDS: a cautionary tale. Trends Mol. Med. 7, 379-381 (2001)

22. Colonna, M., Brooks, E.G., Falco, M., Ferrara, G.B. \& Strominger, J.L. Generation of allospecific natural killer cells by stimulation across a polymorphism of HLA-C. Science 260, 1121-1124 (1993).

23. Boyington, J.C., Brooks, A.G. \& Sun, P.D. Structure of killer cell immunoglobulinlike receptors and their recognition of the class I MHC molecules. Immunol. Rev. 181, 66-78 (2001).

24. Phillips, J.H., Gumperz, J.E., Parham, P. \& Lanier, L.L. Superantigen-dependent, cell-mediated cytotoxicity inhibited by MHC class I receptors on T lymphocytes. Science 268, 403-405 (1995).

25. De Maria, A. et al. Expression of HLA class I-specific inhibitory natural killer cell receptors in HIV-specific cytolytic T lymphocytes: impairment of specific cytolytic functions. Proc. Natl Acad. Sci. USA 94, 10285-10288 (1997).
26. Koup, R.A. et al. Temporal association of cellular immune responses with the initial control of viremia in primary human immunodeficiency virus type 1 syndrome. J. Virol. 68, 4650-4655 (1994).

27. Kelleher, A.D. et al. Clustered mutations in HIV-1 gag are consistently required for escape from HLA-B27-restricted cytotoxic T lymphocyte responses. J. Exp. Med. 193, 375-386 (2001).

28. Hughes, A.L., Yeager, M. \& Carrington, M. Peptide binding function and the paradox of HLA disease associations. Immunol. Cell. Biol. 74, 444-448 (1996).

29. Peruzzi, M., Wagtmann, N. \& Long, E.O. A p70 killer cell inhibitory receptor specific for several HLA-B allotypes discriminates among peptides bound to HLAB*2705. J. Exp. Med. 184, 1585-1590 (1996).

30. Kajiwara, K., Berson, E.L. \& Dryja, T.P. Digenic retinitis pigmentosa due to mutations at the unlinked peripherin/RDS and ROM1 loci. Science 264, 1604-1608 (1994). 